\title{
RESIDUAL VIBRATIONS OF ELECTROSTATIC MICRO-ACTUATORS WITH NOVEL SHAPED CANTILEVERS
}

Ming-Hung Hsu

Department of Electrical Engineering, National Penghu University, Penghu, Taiwan., hsu@npu.edu.tw

Follow this and additional works at: https://jmstt.ntou.edu.tw/journal

Part of the Electrical and Computer Engineering Commons

\section{Recommended Citation}

Hsu, Ming-Hung (2009) "RESIDUAL VIBRATIONS OF ELECTROSTATIC MICRO-ACTUATORS WITH NOVEL SHAPED CANTILEVERS," Journal of Marine Science and Technology. Vol. 17: Iss. 2, Article 2.

DOI: $10.51400 / 2709-6998.1962$

Available at: https://jmstt.ntou.edu.tw/journal/vol17/iss2/2

This Research Article is brought to you for free and open access by Journal of Marine Science and Technology. It has been accepted for inclusion in Journal of Marine Science and Technology by an authorized editor of Journal of Marine Science and Technology. 


\title{
RESIDUAL VIBRATIONS OF ELECTROSTATIC MICRO-ACTUATORS WITH NOVEL SHAPED CANTILEVERS
}

\author{
Ming-Hung Hsu*
}

Key words: Wilson- $\theta$ method, differential quadrature method, electrostatic micro-actuator, microelectromechanical system, nonlinear analysis, Kelvin-Voigt damping, novel shaped cantilever.

\begin{abstract}
This paper subjects electrostatic curved electrode actuators with novel shaped cantilevers to nonlinear dynamic analysis using the differential quadrature method. Numerical results are compared with experimental results to assess the efficiency and systematic procedure of this novel approach for nonlinear differential equations. The pull-in voltages computed for different structures correlate well with published data. Several characteristics of different combinations of shaped cantilevers and curved electrodes are also examined in this paper. Dynamic responses of actuators with novel shapes with several pull-in voltages are determined. The transitional responses for the derived systems are calculated using the Wilson- $\theta$ method.
\end{abstract}

\section{INTRODUCTION}

Microelectromechanical systems, when exploiting microscale effects, have interesting and unique characteristics. Microelectromechanical systems are attracting considerable attention due to their ability to facilitate excellent electronic sensing, actuating, and computing. Different numerical models were proposed by Legtenberg et al. [14, 16, 24, 25] to analyze electrostatically deformed diaphragms. Numerical results suggested that electrostatic deformation calculated using a one-dimensional model is very close to that determined using a complex three-dimensional model. Hirai et al. $[18,19,20]$ considered the deflection characteristics of electrostatic actuators with shaped modified electrodes and cantilevers. Osterberg et al. [31] proposed different numerical models to analyze electrostatically deformed diaphragms. Qiao et al. [32]

Paper submitted 11/05/07; accepted 03/17/08. Author for correspondence: Ming-Hung Hsu (e-mail: hsu@npu.edu.tw).

*Department of Electrical Engineering, National Penghu University, Penghu, Taiwan. presented a design of suspension beam to achieve parallelplate actuator with extended working range based on twolayered polysilicon surface process. This paper presents alternative equations of residual vibrations of electrostatic actuators. The differential quadrature technique is applied to formulate the electrostatic field problems in a matrix form. The integrity and computational accuracy of the differential quadrature method in this problem are demonstrated through various case studies. With the recent development of stable responses and high performance actuator structures, damping enhancement has become a very significant issue. A number of new actuator designs have been proposed for overcoming the pull-in disadvantage. The possible effects of Kelvin-Voigt and external damping coefficients on the micro-actuator are included in the proposed model.

\section{DIFFERENTIAL QUADRATURE METHOD}

The differential quadrature is originally developed by simple analogy with the integral quadrature, which is derived using the interpolation function $[1,2,4]$. The differential quadrature method was first introduced by Bellman et al. [1,2]. Bert et al. [3, 4, 5, 6, 7, 8, 10, 15, 22, 23, 29, 34, 35], who investigated static and free vibration of beams and rectangular plates using the differential quadrature method, proposed the $\delta$ technique. Boundary points are chosen at a small distance in the $\delta$ method. The $\delta$ technique can be applied to the double boundary conditions of plate and beam problems. However, $\delta$ cannot be enlarged for solution accuracy, and solutions oscillate when $\delta$ is excessively small. Bert et al. [3, 4, 5, 6, 7, 8, 10, $15,22,23,29,34,35]$ accounted for boundary conditions in the weighted coefficients. In the formulation of differential quadrature approach, multiple boundary conditions are directly applied to the weighted coefficients and selecting a nearby point, as required when using the $\delta$-interval method, is not necessary. The accuracy of calculated results will be independent of the value of the $\delta$-interval. Liew et al. [13, 17, 26, 27], who analyzed rectangular plates resting on Winkler foundations using the differential quadrature method, also presented a static analysis of laminated composite plates subjected to transverse loads using the differential quadrature method. Malekzadeh and Setoodeh [28] studied the nonlinear 
behavior of symmetric and antisymmetric cross ply, thin to moderately thick, elastic rectangular laminated plates resting on nonlinear elastic foundations using the differential quadrature method. De Rosa and Lippiello [12] dealt with the free vibrations of parallel double-beams joined by a Winkler-type homogeneous elastic foundation using the differential quadrature method. The differential quadrature method is a continuous function that can be approximated by a high-order polynomial in the overall domain, and a derivative of a function at a sample point can be approximated as a weighted linear summation of functional values at all sample points in the overall domain of that variable. Using this approximation, the differential equation is then transformed into a set of algebraic equations. The number of equations depends on the number of sample points selected. Potential oscillations in numerical results arising from high-order polynomials can be eliminated by employing numerical interpolation schemes. For a function, $f(z)$, the differential quadrature method approximation for the $m$-th order derivative at the $i$-th sample point is given as follows [4].

$$
\left.\left.\frac{d^{m}}{d z^{m}}\left\{\begin{array}{c}
f\left(z_{1}\right) \\
f\left(z_{2}\right) \\
\cdot \\
\cdot \\
\cdot \\
f\left(z_{N}\right)
\end{array}\right\} \cong\left[D_{i j}^{(m)}\right]\right\} \begin{array}{c}
f\left(z_{1}\right) \\
f\left(z_{2}\right) \\
\cdot \\
\cdot \\
\cdot \\
f\left(z_{N}\right)
\end{array}\right\} \text { for } i, j=1,2, \ldots, N(1)
$$

where $f\left(z_{i}\right)$ is the functional value at grid point $z_{i}$, and $D_{i j}^{(m)}$ is the weighting coefficient of the $m$-th order differentiation attached to these functional values. For a micro-cantilever structure problem, the most convenient technique is to choose the grid points in a grid point distribution that is equally spaced. Numerical results with poor accuracy were acquired in this study using this equally spaced distribution. This finding demonstrates that the choice of a grid point distribution and test functions markedly influence the efficiency and accuracy of results in some cases. Selection of grid points always has an important role in solution accuracy when using the differential quadrature method. The unequally spaced sample points on each micro-beam using the Chebyshev-Gauss-Lobatto distribution in the present computation are distributed as follows $[4,30]$.

$$
z_{i}=\frac{1}{2}\left(1-\cos \frac{(i-1) \pi}{N-1}\right) \quad \text { for } i=1,2, \ldots, N
$$

Some researchers have shown that the roots of the shifted Chebyshev polynomials in the $(0,1)$ domain can equally produce good results $[4,30]$. The differential quadrature weighted coefficients can be derived using numerous techniques. To overcome the numerically poor conditions when determining the weighted coefficients, $D_{i j}^{(m)}$, a Lagrangian interpolation polynomial was introduced [4], which is as follows.

$$
f(z) \cong \sum_{i=1}^{N} \frac{M(z)}{\left(z-z_{i}\right) M_{1}\left(z_{i}\right)} f\left(z_{i}\right)
$$

where

$$
\begin{gathered}
M(z)=\prod_{j=1}^{N}\left(z-z_{j}\right), \\
M_{1}\left(z_{i}\right)=\prod_{j=1, j \neq i}^{N}\left(z_{i}-z_{j}\right) \quad \text { for } i=1,2, \ldots, N
\end{gathered}
$$

Substituting (3) into (1) yields the following equations [4].

$$
D_{i j}^{(1)}=\frac{M_{1}\left(z_{i}\right)}{\left(z_{i}-z_{j}\right) M_{1}\left(z_{j}\right)} \text { for } i, j=1,2, \ldots, N \text { and } i \neq j(4)
$$

and

$$
D_{i i}^{(1)}=-\sum_{j=1, j \neq i}^{N} D_{i j}^{(1)} \quad \text { for } i=1,2, \ldots, N
$$

Once the grid points are selected, the coefficients of the weighted matrix can be acquired using (4) and (5). Notably, the numbers of test functions exceed the highest order of the derivative in the governing equations. High-order derivatives of weighted coefficients can also be acquired using matrix multiplication [4], which is as follows.

$$
\begin{array}{ll}
D_{i j}^{(2)}=\sum_{k=1}^{N} D_{i k}^{(1)} D_{k j}^{(1)} & \text { for } i, j=1,2, \ldots, N \\
D_{i j}^{(3)}=\sum_{k=1}^{N} D_{i k}^{(1)} D_{k j}^{(2)} & \text { for } i, j=1,2, \ldots, N \\
D_{i j}^{(4)}=\sum_{k=1}^{N} D_{i k}^{(1)} D_{k j}^{(3)} & \text { for } i, j=1,2, \ldots, N
\end{array}
$$

\section{DYNAMIC BEHAVIOR OF MICRO-ACTUATORS}

Figures 1 and 2 show the geometry of an electrostatic actuator. Variable $t_{0}$ is the thickness at the actuator root, $t_{1}$ denotes the tip thickness, and $L$ is the cantilever length.

An electrostatic force, introduced from the applied voltage difference between the curved electrode and cantilever, pulls the cantilever toward to the curved electrode. Shape function $S$ denotes the shape of the curved electrode, and is presented as a polynomial, i.e., $S=\delta_{\max }(z / L)^{n}$, where $\delta_{\max }$ is the tip gap 


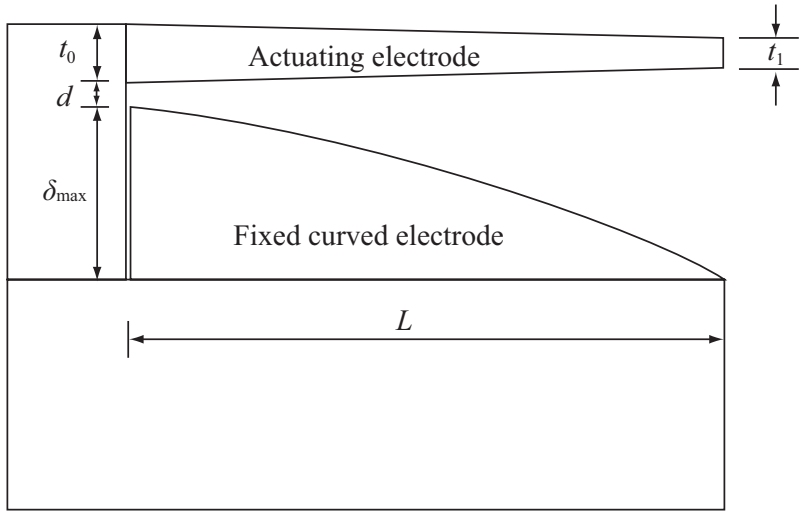

Fig. 1. Schematic of a curved electrode micro-actuator.

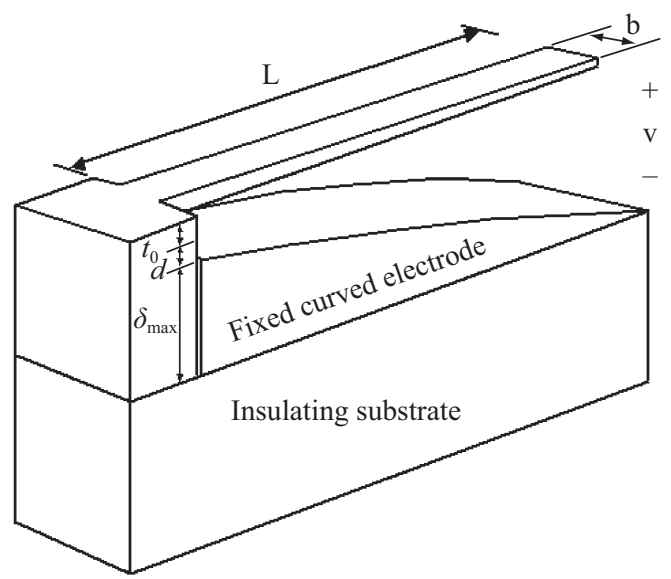

Fig. 2. Schematic of a curved electrode actuator based on microelectromechanical devices.

distance of the curved electrode at $z=L$, and $n$ is the polynomial order of electrode shape. The electrode shape varies with the value of $n$. The distance between the cantilever and curved electrode declines as the polynomial order $n$ of the curve increases. By applying the long beam assumption, the strain energy of the bended cantilever can be simplified as

$$
U=\frac{1}{2} \int_{0}^{L} E I\left(\frac{\frac{\partial^{2} v}{\partial z^{2}}}{\left(1+\left(\frac{\partial v}{\partial z}\right)^{2}\right)^{\frac{3}{2}}}\right)^{2} d z
$$

where $v(z)$ is the deflection of the actuator and $E$ is Young's modulus of the actuator. $I(z)$ is the moment of inertia of cross-sectional area of the micro-actuator, which is $I(z)=$ $I_{0}(1+\beta(z / L))^{3} . \beta$ is defined as the ratio of $\left(t_{1}-t_{0}\right) / t_{0}$ and $I_{0}=$ $b_{0} t_{0}^{3} / 12$. Because

$$
\frac{\frac{\partial^{2} v}{\partial z^{2}}}{\left(1+\left(\frac{\partial v}{\partial z}\right)^{2}\right)^{\frac{3}{2}}} \cong \frac{\partial^{2} v}{\partial z^{2}}
$$

Equation (9) can be written as follows.

$$
U=\frac{1}{2} \int_{0}^{L} E I\left(\frac{\partial^{2} v}{\partial z^{2}}\right)^{2} d z
$$

The electrostatic force per unit length of the micro-actuator is given by the energy differentiation as follows.

$$
q=\frac{1}{2} \bar{V}^{2} \frac{d C}{d y}
$$

where $q$ is the electrostatic force per unit length of the micro-actuator, $C$ is the capacitance per unit length along the cantilever, and $\bar{V}$ is applied voltage. In considering the electrostatic force, virtual work $\delta W_{1}$ is performed by the bended actuator and can be derived as follows.

$$
\delta W_{1}=\int_{0}^{L} \frac{\varepsilon_{0} b \bar{V}^{2}}{2\left(d+S-\frac{\beta z t_{0}}{2 L}-v\right)^{2}} \delta v d z
$$

where $\varepsilon_{0}$ is the dielectric constant in the air, i.e., $\varepsilon_{0}=8.85 \times$ $10^{-12}, b$ is the width of the micro-actuator, and $d$ is the initial gap as shown in Figs. 1 and 2. The dielectric layer also prevents short circuits. The cross-sectional area of the micro-actuator is $A(z)=b_{0} t_{0}(1+\beta z / L)$. The dynamic behavior of the microelectromechanical system is of interest to designers of electrostatic actuators. Actuator residual response can affect the performance of the electrostatic actuator system. However, due to the difficulty associated with the nonlinearlity introduced between actuator deflection and electrostatic force, this residual vibration phenomenon has been examined in very few studies. The effects of electrode shape on residual response have generally not been described. The kinetic energy of the micro-actuator can be derived as

$$
T=\frac{1}{2} \int_{0}^{L} \rho A\left(\frac{\partial v}{\partial t}\right)^{2} d z
$$

where $A(z)$ is the cross-sectional area of the micro-actuator, that is, $A(z)=A_{0}(1+\beta(z / L))$. For generality, Kelvin-Voigt damping and external damping effects have been considered in formulating the equations of motion. External damping is a viscous resistance to transverse displacement of the actuator; KelvinVoigt damping is a viscous resistance to straining of actuator materials $[11,33]$. External damping force $\bar{C}_{e} \frac{\partial v}{\partial t}$ is assumed 
for resistance to actuator transverse velocity. The Kelvin-Voigt damping force $\bar{C}_{i} \frac{\partial^{2}}{\partial z^{2}}\left(E I \frac{\partial^{3} v}{\partial t \partial z^{2}}\right)$ is assumed for resistance to actuator strain velocity. In considering the Kelvin-Voigt and external damping effects in the actuator, virtual work $\delta W_{2}$ done by the actuator for a virtual displacement $\delta v$ can be derived as

$$
\delta W_{2}=-\int_{0}^{L} \bar{C}_{e} \frac{\partial v}{\partial t} \delta v d z-\int_{0}^{L} \bar{C}_{i} \frac{\partial^{2}}{\partial z^{2}}\left(E I \frac{\partial^{3} v}{\partial t \partial z^{2}}\right) \delta v d z
$$

where $\bar{C}_{e}$ and $\bar{C}_{i}$ are external damping and Kelvin-Voigt damping coefficients, respectively. Substituting (11), (13), (14) and (15) into the Hamilton equation yields

$$
\int_{t_{1}}^{t_{2}}\left(\delta T-\delta U+\delta W_{1}+\delta W_{2}\right) d t=0
$$

The equation of motion of the electrostatic micro-actuator can be derived as

$$
\begin{array}{r}
\rho A \frac{\partial^{2} v}{\partial t^{2}}+\bar{C}_{e} \frac{\partial v}{\partial t}+\bar{C}_{i} \frac{\partial^{2}}{\partial z^{2}}\left(E I \frac{\partial^{3} v}{\partial z^{2} \partial t}\right) \\
+\frac{\partial^{2}}{\partial z^{2}}\left(E I \frac{\partial^{2} v}{\partial z^{2}}\right)=\frac{\varepsilon_{0} b \bar{V}^{2}}{2\left(d+S-\frac{\beta z t_{0}}{2 L}-v\right)^{2}}
\end{array}
$$

The dynamic response $v(z, t)$ of an electrostatic micro-actuator is governed by the above fourth-order partial differential equation. The boundary conditions of the clamped-free microbeam are

$$
\begin{gathered}
v(0, t)=0 \\
\frac{\partial v(0, t)}{\partial z}=0 \\
E I \frac{\partial v^{2}(L, t)}{\partial z^{2}}=0 \\
\frac{\partial}{\partial z}\left(E I \frac{\partial v^{2}(L, t)}{\partial z^{2}}\right)=0
\end{gathered}
$$

This nonlinear equation does not have an analytical solution; however, numerical approaches can help solve this equation. In seeking an efficient discretization technique to acquire an accurate numerical solution with very small number of grid points, the differential quadrature method is utilized to solve numerically these nonlinear partial differential equations. By applying the differential quadrature method, Eq. (1) is subs- tituted into (17)-(21). The equations of motion for the microactuator can be discretized in a matrix form with respect to the sample points as follows.

$$
[M]\left\{\frac{\partial^{2} v}{\partial t^{2}}\right\}+[C]\left\{\frac{\partial v}{\partial t}\right\}+[K]\{v\}=\{F\}
$$

The elements in the mass, damping and stiffness matrices are

$$
\begin{gathered}
M_{i i}=0 \text { for } i=1,2 \\
M_{i i}=\rho A \text { for } i=3,4, \ldots, N-2 \\
M_{i i}=0 \text { for } i=N-1, N
\end{gathered}
$$$$
M_{i j}=0 \text { for } i \neq j, i=1,2, \ldots, N \text { and } j=1,2, \ldots, N
$$$$
C_{i j}=0 \text { for } i=1,2, N-1, N \text { and } j=1,2, \ldots, N
$$

$$
\begin{aligned}
C_{i i}= & \bar{C}_{e}+\left.\bar{C}_{i}\left[\frac{\partial^{2}}{\partial z^{2}} E I(z)\right]\right|_{z=z_{i}} \frac{D_{i i}^{(2)}}{L^{2}}+\left.2 \bar{C}_{i}\left[\frac{\partial}{\partial z} E I(z)\right]\right|_{z=z_{i}} \frac{D_{i i}^{(3)}}{L^{3}} \\
& +\bar{C}_{i} E I\left(z_{i}\right) \frac{D_{i i}^{(4)}}{L^{4}} \text { for } i=3,4, \ldots, N-2
\end{aligned}
$$

$$
\begin{gathered}
C_{i j}=\left.\bar{C}_{i}\left[\frac{\partial^{2}}{\partial z^{2}} E I(z)\right]\right|_{z=z_{i}} \frac{D_{i j}^{(2)}}{L^{2}}+\left.2 \bar{C}_{i}\left[\frac{\partial}{\partial z} E I(z)\right]\right|_{z=z_{i}} \frac{D_{i j}^{(3)}}{L^{3}} \\
+\bar{C}_{i} E I\left(z_{i}\right) \frac{D_{i j}^{(4)}}{L^{4}} \\
\text { for } i \neq j, i=3,4, \ldots, N-2 \text { and } j=1,2, \ldots, N \\
\qquad K_{11}=1 \\
K_{2 j}=\frac{D_{1 j}^{(1)}}{L} \quad \text { for } j=2,3, \ldots, N \\
\text { for } \quad j=1,2, \ldots, N
\end{gathered}
$$

$$
\begin{aligned}
K_{i j}= & {\left.\left[\frac{\partial^{2}}{\partial z^{2}} E I(z)\right]\right|_{z=z_{i}} \frac{D_{i j}^{(2)}}{L^{2}}+\left.2\left[\frac{\partial}{\partial z} E I(z)\right]\right|_{z=z_{i}} \frac{D_{i j}^{(3)}}{L^{3}} } \\
& +E I\left(z_{i}\right) \frac{D_{i j}^{(4)}}{L^{4}}
\end{aligned}
$$$$
\text { for } i=3,4, \ldots, N-2 \text { and } j=1,2, \ldots, N
$$ 


$$
\begin{gathered}
K_{N-1, j}=E I\left(z_{N}\right) \frac{D_{N, j}^{(2)}}{L^{2}} \quad \text { for } j=1,2, \ldots, N \\
K_{N, j}=\left.\left[\frac{\partial E I(z)}{\partial z}\right]\right|_{z=z_{N}} \frac{D_{N, j}^{(2)}}{L^{2}}+E I\left(z_{N}\right) \frac{D_{N, j}^{(3)}}{L^{3}} \text { for } \\
j=1,2, \ldots, N \\
F_{i}=\frac{F_{i}=0 \text { for } i=1,2, N-1, N}{2\left(d+S-\frac{\beta z t_{0}}{2 L}-v\right)^{2}} \text { for } i=3,4, \ldots, N-2
\end{gathered}
$$

In this paper, the dynamic responses of grid points on the micro-actuator are solved using the Wilson- $\theta$ approach. The Wilson- $\theta$ method assumes that acceleration terms at sample points vary linearly between two time points. In the Wilson- $\theta$ iteration, the $i$-th time step can be expressed as

$$
t_{i}^{w}=(i-1) \Delta t
$$

The acceleration term is assumed to vary linearly between $t_{i}^{w}$ and $t_{i}^{w}+\theta_{w} \Delta t$. The acceleration term can then be predicted at any time $t_{i}^{w}+\tau$ with $0 \leq \tau \leq \theta_{w} \Delta t$. A number of studies noted that the Wilson- $\theta$ method is unconditionally stable with a factor of $\theta_{w} \geq 1.37[9,11,21,33]$. The acceleration vector of sample points at time $t_{i}^{w}+\tau$ can be approximated as

$$
\begin{aligned}
& \left\{\frac{\partial^{2} v\left(z, t_{i}^{w}+\tau\right)}{\partial t^{2}}\right\}=\left\{\frac{\partial^{2} v\left(z, t_{i}^{w}\right)}{\partial t^{2}}\right\} \\
& +\frac{\tau}{\theta_{w} \Delta t}\left\{\left\{\frac{\partial^{2} v\left(z, t_{i}^{w}+\theta_{w} \Delta t\right)}{\partial t^{2}}\right\}-\left\{\frac{\partial^{2} v\left(z, t_{i}^{w}\right)}{\partial t^{2}}\right\}\right\}
\end{aligned}
$$

where subscript $i$ denotes the $i$-th step and $\Delta t$ is the time increment. Similarly, the velocity and displacement vectors of sample points at time $t_{i}^{w}+\tau$ can be approximated using following two equations, respectively.

$$
\begin{gathered}
\left\{\frac{\partial v\left(z, t_{i}^{w}+\tau\right)}{\partial t}\right\}=\left\{\frac{\partial v\left(z, t_{i}^{w}\right)}{\partial t}\right\}+\left\{\frac{\partial^{2} v\left(z, t_{i}^{w}\right)}{\partial t^{2}}\right\} \tau \\
+\frac{\tau^{2}}{2 \theta_{w} \Delta t}\left\{\left\{\frac{\partial^{2} v\left(z, t_{i}^{w}+\theta_{w} \Delta t\right)}{\partial t^{2}}\right\}-\left\{\frac{\partial^{2} v\left(z, t_{i}^{w}\right)}{\partial t^{2}}\right\}\right\}
\end{gathered}
$$

and

$$
\begin{aligned}
& \left\{v\left(z, t_{i}^{w}+\tau\right)\right\}=\left\{v\left(z, t_{i}^{w}\right)\right\}+\left\{\frac{\partial v\left(z, t_{i}^{w}\right)}{\partial t}\right\} \tau \\
& +\frac{1}{2}\left\{\frac{\partial^{2} v\left(z, t_{i}^{w}\right)}{\partial t^{2}}\right\} \tau^{2} \\
& +\frac{\tau^{3}}{6 \theta_{w} \Delta t}\left\{\left\{\frac{\partial^{2} v\left(z, t_{i}^{w}+\theta_{w} \Delta t\right)}{\partial t^{2}}\right\}-\left\{\frac{\partial^{2} v\left(z, t_{i}^{w}\right)}{\partial t^{2}}\right\}\right\}
\end{aligned}
$$

Substituting $\tau=\theta_{w} \Delta t$ into (40), the velocity vector can be rewritten as

$$
\begin{aligned}
& \left\{\frac{\partial v\left(z, t_{i}^{w}+\theta_{w} \Delta t\right)}{\partial t}\right\}=\left\{\frac{\partial v\left(z, t_{i}^{w}\right)}{\partial t}\right\} \\
& +\frac{\theta_{w} \Delta t}{2}\left\{\left\{\frac{\partial^{2} v\left(z, t_{i}^{w}+\theta_{w} \Delta t\right)}{\partial t^{2}}\right\}+\left\{\frac{\partial^{2} v\left(z, t_{i}^{w}\right)}{\partial t^{2}}\right\}\right\}
\end{aligned}
$$

Similarly, the displacement vector of grid points can then be rewritten as

$$
\begin{gathered}
\left\{v\left(z, t_{i}^{w}+\theta_{w} \Delta t\right)\right\}=\left\{v\left(z, t_{i}^{w}\right)\right\}+\theta_{w} \Delta t\left\{\frac{\partial v\left(z, t_{i}^{w}\right)}{\partial t}\right\} \\
+\frac{\theta_{w}^{2}(\Delta t)^{2}}{6}\left\{\left\{\frac{\partial^{2} v\left(z, t_{i}^{w}+\theta_{w} \Delta t\right)}{\partial t^{2}}\right\}+2\left\{\frac{\partial^{2} v\left(z, t_{i}^{w}\right)}{\partial t^{2}}\right\}\right\}
\end{gathered}
$$

Equation (39) can be solved to obtain

$$
\begin{aligned}
& \left\{\frac{\partial^{2} v\left(z, t_{i}^{w}+\theta_{w} \Delta t\right)}{\partial t^{2}}\right\} \\
& =\frac{6}{\theta_{w}^{2}(\Delta t)^{2}}\left\{\left\{v\left(z, t_{i}^{w}+\theta_{w} \Delta t\right)\right\}-\left\{v\left(z, t_{i}^{w}\right)\right\}\right\} \\
& -\frac{6}{\theta_{w} \Delta t}\left\{\frac{\partial v\left(z, t_{i}^{w}\right)}{\partial t}\right\}-2\left\{\frac{\partial^{2} v\left(z, t_{i}^{w}\right)}{\partial t^{2}}\right\}
\end{aligned}
$$

Substituting (44) into (42), the velocity vector at time $t_{i}^{w}+$ $\theta_{w} \Delta t$ can be written as

$$
\begin{gathered}
\left\{\frac{\partial v\left(z, t_{i}^{w}+\theta_{w} \Delta t\right)}{\partial t}\right\}=\frac{3}{\theta_{w} \Delta t}\left\{\left\{v\left(z, t_{i}^{w}+\theta_{w} \Delta t\right)\right\}-\left\{v\left(z, t_{i}^{w}\right)\right\}\right\} \\
-2\left\{\frac{\partial v\left(z, t_{i}^{w}\right)}{\partial t}\right\}-\frac{\theta_{w} \Delta t}{2}\left\{\frac{\partial^{2} v\left(z, t_{i}^{w}\right)}{\partial t^{2}}\right\}
\end{gathered}
$$




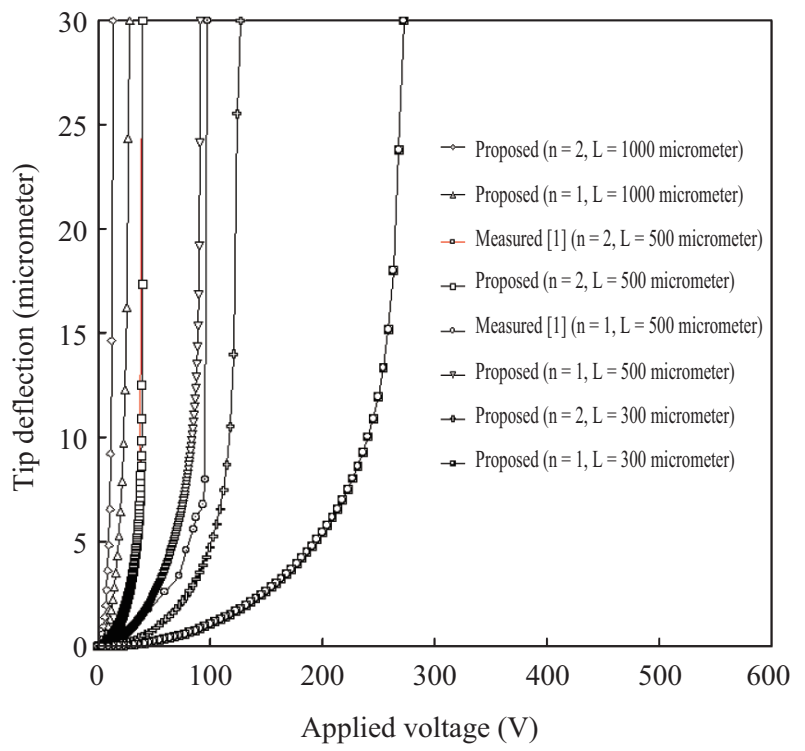

Fig. 3. Comparisons of tip deflections of an actuating electrode with different applied voltages and electrode shapes. Proposed results solved using the differential quadrature method.

The force vector at time $t_{i}^{w}+\theta_{w} \Delta t$ can be approximated as

$$
\begin{array}{r}
\left\{F\left(z, t_{i}^{w}+\theta_{w} \Delta t\right)\right\}=\left\{F\left(z, t_{i}^{w}\right)\right\} \\
+\theta_{w}\left\{\left\{F\left(z, t_{i+1}^{w}\right)\right\}-\left\{F\left(z, t_{i}^{w}\right)\right\}\right\}
\end{array}
$$

Substituting (43)-(46) into (22) yields the following equation.

$$
\begin{gathered}
{\left[\frac{6}{\theta_{w}^{2}(\Delta t)^{2}}[M]+\frac{3}{\theta_{w} \Delta t}[C]+[K]\left\{v\left(z, t_{i}^{w}+\theta_{w} \Delta t\right)\right\}\right.} \\
\quad=\left\{F\left(z, t_{i}^{w}\right)\right\}+\theta_{w}\left\{\left\{F\left(z, t_{i+1}^{w}\right)\right\}-\left\{F\left(z, t_{i}^{w}\right)\right\}\right\} \\
+\left[\frac{6}{\theta_{w}(\Delta t)^{2}}[M]+\frac{3}{\theta_{w} \Delta t}[C]\right]\left\{v\left(z, t_{i}^{w}\right)\right\} \\
+\left[\frac{6}{\theta_{w} \Delta t}[M]+2[C]\right]\left\{\frac{\partial v\left(z, t_{i}^{w}\right)}{\partial t}\right\} \\
+\left[2[M]+\frac{\theta_{w} \Delta t}{2}[C]\right]\left\{\frac{\partial^{2} v\left(z, t_{i}^{w}\right)}{\partial t^{2}}\right\}
\end{gathered}
$$

The acceleration vector of sample points at time $t_{i+1}^{w}$ can be expressed as

$$
\begin{gathered}
\left\{\frac{\partial^{2} v\left(z, t_{i+1}^{w}\right)}{\partial t^{2}}\right\}=\frac{6}{\theta_{w}^{3}(\Delta t)^{2}}\left\{\left\{v\left(z, t_{i}^{w}+\theta_{w} \Delta t\right)\right\}-\left\{v\left(z, t_{i}^{w}\right)\right\}\right\} \\
-\frac{6}{\theta_{w}^{2} \Delta t}\left\{\frac{\partial v\left(z, t_{i}^{w}\right)}{\partial t}\right\}+\left(1-\frac{3}{\theta_{w}}\right)\left\{\frac{\partial^{2} v\left(z, t_{i}^{w}\right)}{\partial t^{2}}\right\}
\end{gathered}
$$

Similarly, the velocity and displacement vectors of sample points at time $t_{i+1}^{w}$ can be approximated as

$$
\begin{aligned}
& \left\{\frac{\partial v\left(z, t_{i+1}^{w}\right)}{\partial t}\right\}=\left\{\frac{\partial v\left(z, t_{i}^{w}\right)}{\partial t}\right\} \\
& +\frac{\Delta t}{2}\left\{\left\{\frac{\partial^{2} v\left(z, t_{i+1}^{w}\right)}{\partial t^{2}}\right\}-\left\{\frac{\partial^{2} v\left(z, t_{i}^{w}\right)}{\partial t^{2}}\right\}\right\}
\end{aligned}
$$

and

$$
\begin{gathered}
\left\{v\left(z, t_{i+1}^{w}\right)\right\}=\left\{v\left(z, t_{i}^{w}\right)\right\}+\Delta t\left\{\frac{\partial v\left(z, t_{i}^{w}\right)}{\partial t}\right\} \\
+\frac{(\Delta t)^{2}}{6}\left\{\left\{\frac{\partial^{2} v\left(z, t_{i+1}^{w}\right)}{\partial t^{2}}\right\}+2\left\{\frac{\partial^{2} v\left(z, t_{i}^{w}\right)}{\partial t^{2}}\right\}\right\}
\end{gathered}
$$

\section{NUMERICAL RESULTS AND DISCUSSION}

Figure 3 presents the difference between the calculated and measured tip deflections with different driving voltages, cantilever lengths and electrode shapes. The micro-actuator is made of polysilicon. The material and the geometric parameters of the actuator are as follows: $E=150 \mathrm{GPa}, \delta_{\max }=30 \mu \mathrm{m}$, $b=5 \mu \mathrm{m}, t_{0}=2 \mu \mathrm{m}, d=2 \mu \mathrm{m}$, and $\beta=0$. The differential quadrature approach is programmed in the MATLAB software package. Numerical and measured results reveal that tip deflections calculated using the proposed differential quadrature method are in good agreement with published experimental results [25]. The accuracy of calculated results indicates that the model derived using the differential quadrature method is effective for assessing the deflection of an electrostatic actuator system. Electrode shapes and lengths of the novel cantilever influence the pull-in range. Figure 4 presents the tip responses of the actuator with various electrode forms. The material and geometric parameters of the electrostatic actuator system are as follows: $E=150 \mathrm{GPa}, \rho=2.3 \times 10^{3} \mathrm{~kg} / \mathrm{m}^{3}$, $\bar{C}_{e}=0, \bar{C}_{i}=0, \delta_{\max }=30 \mu \mathrm{m}, b=5 \mu \mathrm{m}, t_{0}=2 \mu \mathrm{m}, d=2 \mu \mathrm{m}$, $L=500 \mu \mathrm{m}$, and $\bar{V}=10 \mathrm{~V}$. Electrode shapes vary with different values for $n$. The nonlinear dynamic equation generated using the proposed differential quadrature method is solved using the Wilson- $\theta$ approach. In this case, the Wilson- $\theta$ method is used with $\theta_{w}=1.4$ and $\Delta t=0.003 \mathrm{~ms}$. 


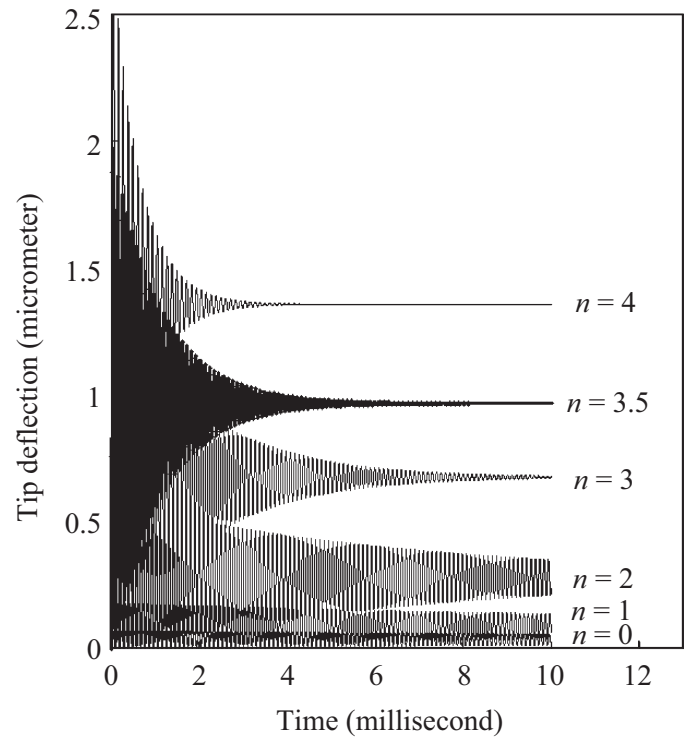

Fig. 4. Tip responses of the micro-actuator with various electrode forms.

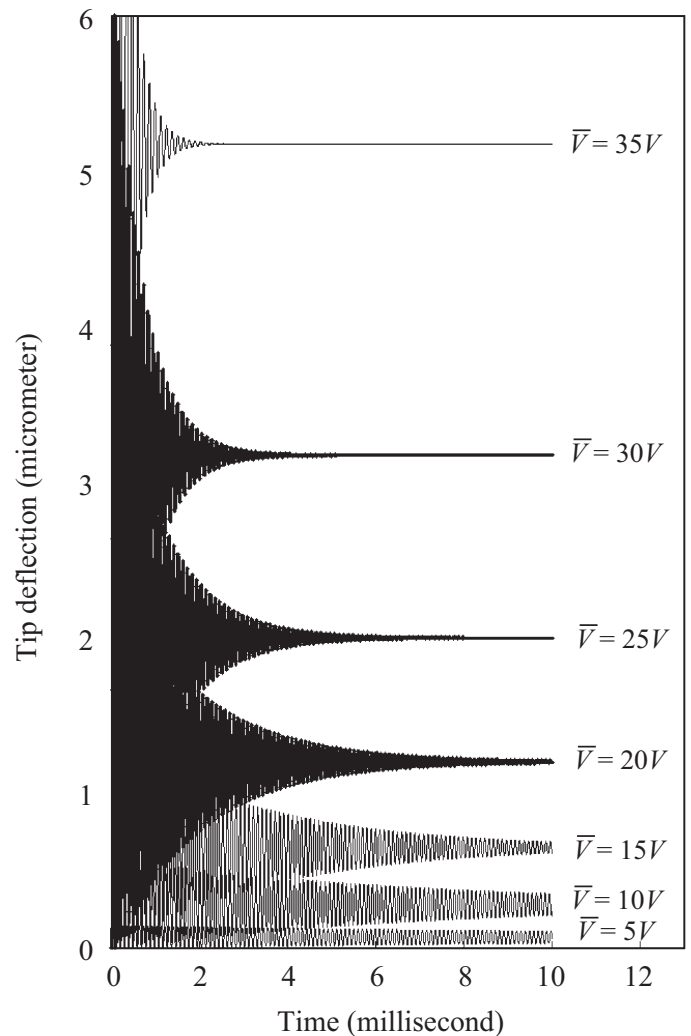

Fig. 5. Tip responses of the micro-actuator with different applied voltages.

Numerical results in Fig. 4 demonstrate that electrode shape markedly affects actuator deflection, and residual vibra- tion. Calculated results demonstrate that tip residual vibration can be decreased considerably as electrode shape with a value of $n$ near 4.

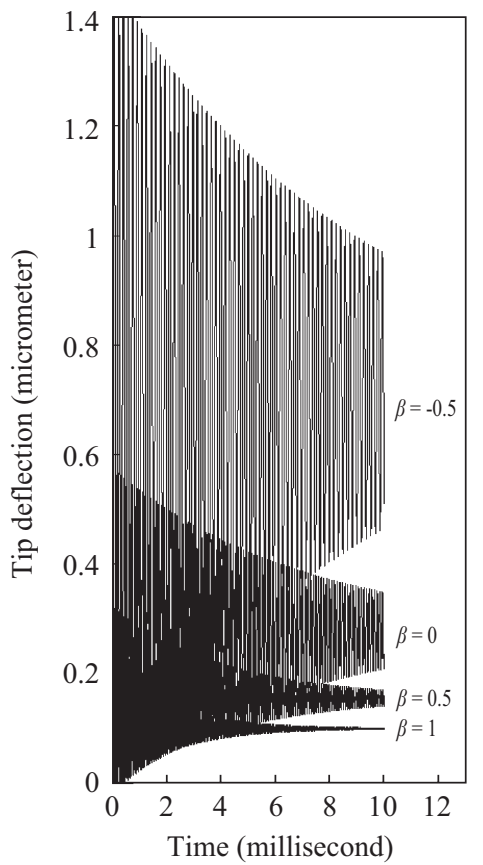

Fig. 6. Tip responses of the micro-actuator with different $\beta$ values.

Figure 5 presents the variation of actuator tip deflection and tip residual vibration after applying different driving voltages. Numerical results in this example demonstrate that driving voltage affects significantly the dynamic behavior of the actuator system. Calculation results also indicate that high driving voltage applied between electrodes always introduces large actuator deflection and serious residual vibration.

Figure 6 presents the variation of tip displacement responses for a micro-actuator with different $\beta$. Numerical results dem- onstrate that the actuator stiffness is enhanced when the value of $\beta$ is large.

Figure 7 shows the variations of actuator tip response with different external damping values. Numerical results demonstrate that the external damping coefficient is an extremely sensitive parameter to actuator residual vibration. Substantial residual vibration is acquired for the system with a zero external damping coefficient.

A similar reduction effect for actuator residual vibration, as shown in Fig. 8, was found for the system with different Kelvin-Voigt damping values. An external damping coefficient of zero is assumed in this case. However, numerical results also indicate that the Kelvin-Voigt damping is as sensitive to residual vibrations as $\beta$ and external damping.

\section{CONCLUSIONS}

Simulation results verify that the differential quadrature method obtains accurate results with relatively minimal computational and modeling efforts. The effects of electrode shape and cantilever beam shape on the pull-in behavior and residual vibration of cantilever actuators are investigated. The 

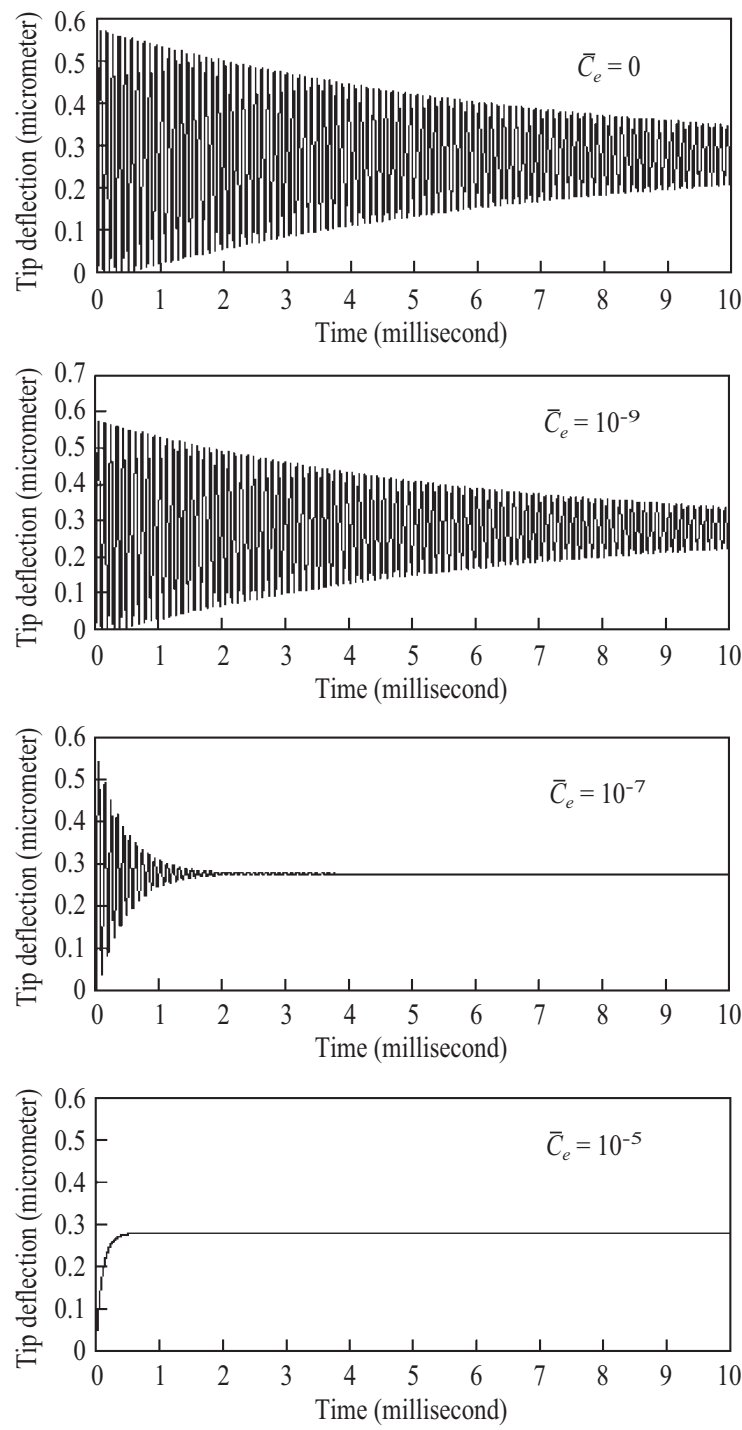

Fig. 7. Variation of tip responses of the micro-actuator with different external damping coefficients.

damping constant of the system has a marked effect on the system's dynamic behavior. Excellent agreements are obtained between measured deflections and deflections solved using the differential quadrature method.

\section{REFERENCES}

1. Bellman, R. E. and Casti, J., "Differential quadrature and long-term integration," Journal of Mathematical Analysis and Application, Vol. 34, pp. 235-238 (1971).

2. Bellman, R. E., Kashef, B. G., and Casti, J., "Differential quadrature: A technique for rapid solution of nonlinear partial differential equations," Journal Computational Physics, Vol. 10, pp. 40-52 (1972).

3. Bert, C. W., Jang, S. K., and Striz, A. G., "Two new approximate methods foranalyzing free vibration of structural components," AIAA Journal, Vol. 26, pp. 612-618 (1988).

4. Bert, C. W. and Mailk, M., "Differential quadrature method in computational mechanics: A review," Applied Mechanics Review, Vol. 49, pp. 1-28 (1996).
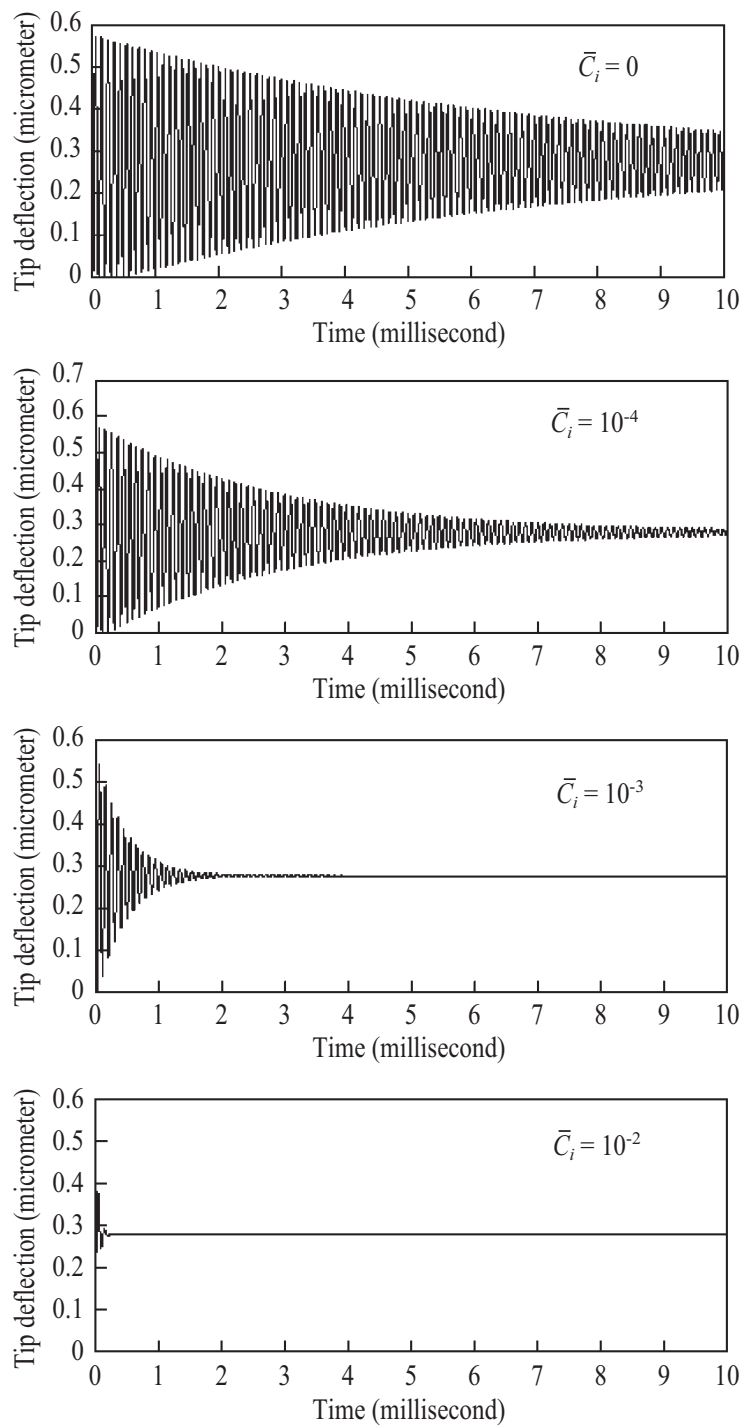

Fig. 8. Tip responses of the micro-actuator with various Kelvin-Voigt damping coefficients.

5. Bert, C. W. and Malik, M., "Free vibration analysis of tapered rectangular plates by differential quadrature method: A semi-analytical approach," Journal of Sound and Vibration, Vol. 190, pp. 41-63 (1996).

6. Bert, C. W. and Malik, M., "On the relative effects of transverse shear deformation and rotary inertia on the free vibration of symmetric cross-ply laminated plates," Journal of Sound and Vibration, Vol. 193, pp. 927-933 (1996).

7. Bert, C. W., Wang, X., and Striz, A. G., "Differential quadrature for static and free vibration analysis of anisotropic plates," International Journal of Solids and Structures, Vol. 30, pp. 1737-1744 (1993).

8. Bert, C. W., Wang, X., and Striz, A. G., "Convergence of the DQ method in the analysis of anisotropic plates," Journal of Sound and Vibration, Vol. 170, pp. 140-144 (1994).

9. Burnett, D. S., Finite Element Analysis, Addison-Wesley Publishing Company (1987).

10. Chen, W. L., Striz, A. G., and Bert, C. W., "High-accuracy plane stress and plate elements in the quadrature element method," International Journal of Solid and Structures, Vol. 37, pp. 627-647 (2000).

11. Clough, R. W. and Penzien, J., Dynamics of Structures, McGraw-Hill (1975). 
12. De Rosa, M. A. and Lippiello, M., "Non-classical boundary conditions and DQM for double-beams," Mechanics Research Communications, Vol. 34, pp. 538-544 (2007).

13. Du, H., Liew, K. M., and Lim, M. K., "Generalized differential quadrature method for buckling analysis," Journal of Engineering Mechanics, Vol. 122, pp. 95-100 (1996).

14. Etwenspock, M., Weustink, M., and Legtenberg, R., "Static and dynamic properties of active joints," The 8-th International Conference on Solid-State Sensors and Actuators, pp. $412-415$ (1995).

15. Feng, Y. and Bert, C. W., "Application of the quadrature method to flexural vibration analysis of a geometrically nonlinear beam," Nonlinear Dynamics, Vol. 3, pp. 13-18 (1992).

16. Gilbert, J. R., Legtenberg, R., and Senturia, S. D., "3D coupled electro-mechanics for MEMS: Application of CoSolve-EM," Proceedings of IEEE Conference on Micro Electro Mechanical System, pp. 122-127 (1995).

17. Han, J. B. and Liew, K. M., "Axisymmetric free vibration of thick annular plates," International Journal of Mechanical Science, Vol. 41, pp. 1089-1109 (1999).

18. Hirai, Y., Marushima, Y., Nishikawa, K., and Tanaka, Y., "Young's modulus evaluation of Si thin film fabricated by compatiable process with Si MEMSs," Microprocesses and Nanotechnology Conference, pp. 82-83 (2002)

19. Hirai, Y., Marushima, Y., and Soda, S., "Electrostatic actuator with novel shaped cantilever," Proceedings of Internal Symposium on Micromechatronics and Human Science, pp. 223-227 (2000).

20. Hirai, Y., Shindo, M., and Tanaka, Y., "Study of large bending and low voltage drive electrostatic actuator with novel shaped cantilever and electrode," Proceedings of Internal Symposium on Micromechatronics and Human Science, pp. 161-164 (1998).

21. Huebner, K. H. and Thrnton, E. A., The Finite Element Method for Engineers, John Wiley \& Sons (1982).

22. Jang, S. K., Bert, C. W., and Striz, A. G., "Application of differential quadrature to static analysis of structural components," International Journal for Numerical Methods in Engineering, Vol. 28, pp. 561-577 (1989).

23. Kang, K., Bert, C. W., and Striz A. G., "Static analysis of a curved shaft subjected to end torques," International Journal of Solids and Structures
Vol. 33, pp. 1587-1596 (1996).

24. Legtenberg, R., Berenschot, E., Elwenspoek, M., and Fluitman, J., "Electrostatic curved electrode actuators," Proceedings of IEEE Conference on Micro Electro Mechanical Systems, pp. 37-42 (1995).

25. Legtenberg, R., Gilbert Senturia, J. S. D., and Elwenspoek, M., "Electrostatic curved electrode actuators," Journal of Microelectromechanical Systems, Vol. 6, pp. 257-265 (1997).

26. Liew, K. M., Han, J. B., and Xiao, Z. M., "Differential quadrature method for thick symmetric cross-ply laminates with first-order shear flexibility," International Journal of Solids and Structures, Vol. 33, pp. 2647-2658 (1996).

27. Liew, K. M., Han, J. B., Xiao, Z. M., and Du, H., "Differential quadrature method for mindlin plates on winkler foundations," International Journal of Mechanical Sciences, Vol. 38, pp. 405-421 (1996).

28. Malekzadeh, P. and Setoodeh, A. R., "Large deformation analysis of moderately thick laminated plates on nonlinear elastic foundations by DQM," Composite Structures, Vol. 80, pp. 569-579 (2007).

29. Malik, M. and Bert, C. W., "Implementing multiple boundary conditions in the DQ solution of higher-order PDE's application to free vibration of plates," International Journal for Numerical Methods in Engineering, Vol. 39, pp. 1237-1258 (1996).

30. Moradi, S. and Taheri, F., "Delamination buckling analysis of general laminated composite beams by differential quadrature method," Composites Part B: Engineering, Vol. 30, pp. 503-511 (1999).

31. Osterberg, P., Yie, H., Cai, X., White, J., and Senturia, S., "Self-conistent simulation and modeling of electrostatically deformed diaphragms," Proceedings of IEEE Conference on Micro Electro Mechanical Systems, pp. 28-32 (1994).

32. Qiao, D. Y., Yuan, W. Z., and Li, X. Y., "A two-beam method for extending the working range of electrostatic parallel-plate micro-actuators," Journal of Electrostatic, Vol. 65, pp. 256-262 (2007).

33. Rao, S. S., Mechanical Vibrations, Addison-Wesley (1990).

34. Striz, A. G., Chen, W., and Bert, C. W., "Static analysis of structures by the quadrature element method (QEM)," International Journal of Solids and Structures, Vol. 31, pp. 2807-2818 (1994).

35. Wang, X. and Bert, C. W., "A new approach in applying differential quadrature to static and free vibrational analyses of beams and plates," Journal of Sound and Vibration, Vol. 162, pp. 566-572 (1993). 